\title{
Dynamics determines Geometry
}

\author{
Sergio A. Hojman* \\ Departamento de Ciencias, Facultad de Artes Liberales, \\ Facultad de Ingeniería y Ciencias, Universidad Adolfo Ibáñez, Santiago, Chile, \\ and Departamento de Física, Facultad de Ciencias, Universidad de Chile, Santiago, Chile, \\ and Centro de Recursos Educativos Avanzados, CREA, Santiago, Chile. \\ J. Gambod \\ Departamento de Física, Universidad de Santiago de Chile, \\ Casilla 30\%, Santiago, Chile \\ and \\ Facultad de Física, \\ Pontificia Universidad Católica de Chile, Santiago, Chile \\ F. Méndezt团 \\ Departamento de Física, Universidad de Santiago de Chile, Casilla 30\%, Santiago, Chile
}

\begin{abstract}
The inverse problem of calculus of variations and $s$-equivalence are re-examined by using results obtained from non-commutative geometry ideas. The role played by the structure of the modified Poisson brackets is discussed in a general context and it is argued that classical $s$-equivalent systems may be non-equivalent at the quantum mechanical level. This last fact is explicitly discussed comparing different approaches to deal with the Nair-Polychronakos oscillator.
\end{abstract}

\section{INTRODUCTION}

Consider a system $S$ of $n$ differential equations for $n$ variables $q^{i}$. The Inverse Problem of the Calculus of Variations (IPCV) deals with the question of existence and uniqueness of variational principles from which the system $S$ may be derived.

If at least one Lagrangian for system $S$ exists then the task of constructing one (or several) variational principle(s) or Lagrangian(s) is also part of the IPCV. This means that variation of the action constructed from one of those Lagrangian functions, yields the original system $S$ or a system $S^{\prime}$ equivalent to it, in the sense that the space of solutions of $S$ and $S^{\prime}$ are identical. If this is the case, systems $S$ and $S^{\prime}$ are called "solution equivalent" or "s-equivalent".

As far as we are aware, the first significant contributions to this field were made by Helmholtz in 1887 [1] and by Darboux in 1894 [2] for second order differential equations. Helmholtz found the conditions for the existence of a Lagrangian written in terms of the (second order) differential equations of the system $S$. If Helmholtz conditions are satisfied, then a Lagrangian which (upon variation of its action integral) yields (exactly) the second order differential equations of system $S$ exists.

A little bit later, Darboux, solved completely the one dimensional $(n=1)$ case showing, in so doing, that in one dimension a Lagrangian (for one second order differential equation) always exists and it is not unique in a non trivial fashion.

\footnotetext{
* sergio.hojman@uai.cl

$\dagger$ jgamboa55@gmail.com

$\ddagger$ fernando.mendez.f@gmail.com
}

There is, of course, the familiar non uniqueness of Lagrangian functions which stems from the addition of a total time derivative of an arbitrary function. In the one dimensional case, there are Lagrangian functions which give rise to (infinitely many) systems of second order differential equations which are s-equivalent (but not identical) to system $S$.

These variational principles give rise to different sets of second order differential equations which have the same set of solutions. The (first order differential equations) Hamiltonian theories constructed from these Lagrangian formulations are different from each other in the sense that they give rise to different Hamiltonian functions and different Poisson Brackets relations.

In 1941, Douglas [3] solved the two dimensional case completely. Three possible outcomes arise in the two dimensional case: a) no Lagrangian exists, b) there is exactly one (up to addition of a total time derivative) Lagrangian or c) there are infinitely many Lagrangian functions for the system of two second order differential equations.

In the case of first order differential equations, Havas [4] made progress towards the solution of this problem which was completely solved by Hojman and Urrutia in 1981 [5], who provided a way of constructing infinitely many Lagrangians for such systems and presented examples of first order Lagrangians for systems for which no second order Lagrangians exist (in this context see also [6], 7]).

It is then clear that the quantization of such systems might give rise to different quantum theorie 1 - not only

\footnotetext{
${ }^{1}$ In this paper we will discuss the first quantization scheme only.
} 
due to the well known problems of operator ordering since different Hamiltonian (and Poisson Brackets) structures give rise to the same classical equations of motion. These Hamiltonian structures cannot be related by canonical transformations.

One could argue that at the end, among all these Hamiltonian structures which, in principle, might give rise to different quantum theories, only those whose predictions are realized in nature - and verified through experiments - are of physical interest. This is true, but here, an implicit assumption is made: nature selects only one (out of infinitely many, in some cases) Hamiltonian structure and discards the rest of them.

A counterexample of this can be found in noncommutative spaces which have attracted much attention recently. Its connection with different problems in physics have been widely discussed in the literature, in the context of string theory [8], field theory [13] and gravitation [14].

Noncommutative quantum mechanics (NCQM), on the other hand, has also been explored as a possible scenario to test the physical consequences of this new structure proposed for space [15]. The two dimensional case, for instance, can be solved for any central potential [16], and it is possible to show that this problem is connected with the Landau problem for the lowest energy levels.

From a different point of view, NCQM has proven to be good laboratory to test new approaches which might shed light on long standing problems in physics. In [17], for instance, a new kind of noncommutativity which incorporates the spin of the particles has been proposed as an alternative mechanism to explain superconductivity involving triplet states. In a recent work [18], its relation with the physics of graphene is explored.

In the present paper we consider a particle whose classical equations of motion (EoM) are a generalization of the EoM of a particle in a magnetic field. We construct two Hamiltonian structures for these classical EoM which give rise to two different and inequivalent quantum theories. One of them is related to the Nair-Polychronakos anisotropic harmonic oscillator [19], while the other one is described by a Hamiltonian which is the addition of a harmonic oscillator Hamiltonian plus a term proportional to angular momentum.

A classical set of second order equations of motion $S$ is said to be non Lagrangian if there exists no Lagrangian which yields second order equations which are (identical or at least) equivalent to the set $S$. Nevertheless, a Hamiltonian structure which produces first order equations equivalent to $S$ may always be constructed using different approaches [5], 7], 22]. The Hamiltonian structures for non Lagrangian systems are always noncommutative in the sense that the space coordinates Poisson Brackets sub-matrix does not vanish [6], [7]. We illustrate this point with some examples.

In order to prove the previous assertions, we will briefly review the construction of first order Lagrangian, Poisson
Brackets and Hamiltonian structures in the last part of this section. In section II, we explore different first order and second order Lagrangian structures that give rise to equivalent sets of equations of motion and their relation to noncommutative spaces. Section III is devoted to the quantum mechanical discussion of the model and, in the last section, discussion and conclusions are presented.

Let us start by considering a set of coordinates in phase space $\left\{x^{a}\right\}$, with $a \in\{1,2, \cdots, 2 n\}$. A first order Lagrangian $L$ is the most general Lagrangian such that its Euler-Lagrange equation are first order, namely [5]

$$
L=\ell_{a}\left(x^{b}\right) \dot{x}^{a}+\ell_{0}\left(x^{b}\right) .
$$

In fact, its (first order) Euler Lagrange equations are

$$
\sigma_{a b} \dot{x}^{b}=\ell_{0, b},
$$

where $A,{ }_{c}$ denotes partial derivative of $A$ with respect to $x^{c}$ and

$$
\sigma_{a b} \equiv \ell_{a, b}-\ell_{b, a} .
$$

The exterior derivative of the Lagrange Brackets twoform $\sigma$ vanishes identically, i.e.,

$$
\sigma_{a b, c}+\sigma_{b c, a}+\sigma_{c a, b} \equiv 0 .
$$

The Hamiltonian is straightforwardly computed. In fact, let us define the Poisson Brackets matrix $\mathbf{J}$ as the inverse (up to a sign) of the Lagrange brackets $\sigma_{a b}$. The first order equations of motion can be also written as

$$
\dot{x}^{a}=\mathbf{J}^{a b} \frac{\partial H}{\partial x^{b}}, \quad \sigma_{a b} \mathbf{J}^{b c}=-\delta_{a}^{c}
$$

where $H$ is the Hamiltonian of the system $\left(H=-\ell_{0}\right)$.

The previous equations may be rewritten as

$$
\dot{x}^{a}=\left[x^{a}, H\right], \quad \text { with } \quad[A, B]=\partial_{a} A \mathbf{J}^{a b} \partial_{b} B,
$$

where $[A, B]$ are the Poisson Brackets relations for any two dynamical variables $A\left(x^{a}\right), B\left(x^{b}\right)$.

Consider a first order system defined by

$$
\dot{x}^{a}=f^{a}\left(x^{b}\right) .
$$

A Hamiltonian structure for it is defined in terms of a Hamiltonian $H$ and a Poisson Brackets matrix $\mathbf{J}$ such that $\mathbf{J}$ is antisymmetric

$$
\mathbf{J}^{a b}=-\mathbf{J}^{b a},
$$

it satisfies Jacobi Identity

$$
\mathbf{J}^{a b}{ }_{d} \mathbf{J}^{d c}+\mathbf{J}^{b c}{ }_{d} \mathbf{J}^{d a}+\mathbf{J}^{c a}{ }_{, d} \mathbf{J}^{d b} \equiv 0,
$$

\footnotetext{
2 We consider an even dimensional phase space for simplicity, which does not mean that odd dimensional phase spaces cannot be defined.
} 
and generates the equations of motion, in conjunction with $H$

$$
f^{a}=\mathbf{J}^{a b} \frac{\partial H}{\partial x^{b}}
$$

Given a Hamiltonian structure for a first order system then the first order Lagrangian may be easily constructed [5], Appendix A of [9], [10], [11].

By the same token, given a second order Lagrangian for a dynamical system, a first order one may be easily constructed (the one which gives rise to Hamilton's equations, for instance).

Nevertheless, the converse is not true, i.e., given a first order Lagrangian it is not always possible to construct a second order Lagrangian for a given dynamical system [5], 6], 7].

In the next section we will specify the set of classical equations of motion we are interested in and then, we will show explicitly two different Lagrangian and Hamiltonian structures for them and their quantum theories.

\section{CLASSICAL EQUATIONS OF MOTION AND LAGRANGIAN STRUCTURES}

Consider a system with $n$ spatial coordinates $\left\{q_{i}\right\}_{i \in\{1,2, \ldots, n\}}$ and the Lagrangian

$$
L_{q \dot{q}}=\frac{1}{2}\left(T_{i j} \dot{q}^{i} \dot{q}^{j}+\theta_{i j} q^{i} \dot{q}^{j}-V_{i j} q^{i} q^{j}\right)
$$

where $T_{i j}, \theta_{i j}$ and $V_{i j}$ are constant matrices with the following symmetry properties

$$
T_{i j}=T_{j i}, \quad \theta_{i j}=-\theta_{j i}, \quad V_{i j}=V_{j i} .
$$

The Euler Lagrange equations are

$$
T_{i j} \ddot{q}^{j}-\theta_{i j} \dot{q}^{j}+V_{i j} q^{j}=0 .
$$

Take, for example $n=2, T_{i j}=m \delta_{i j}, \theta_{i j}=\frac{e}{c} \epsilon_{i j} B$ and $V_{i j}=0$, equation (12) describes the dynamics of a particle of mass $m$ and electrical charge $e$ in the presence of a constant magnetic field $B$ orthogonal to the plane.

A first order Lagrangian may be straightforwardly constructed from this second order Lagrangian (see, for instance Appendix A of [9], [10] and [11]). Define the variables $u^{i}$ by $u^{i} \equiv \dot{q}^{i}$, and the function $\bar{L}_{q u}$ (using the $u^{i}$ definition into (11)) by

$$
\bar{L}_{q u}=\frac{1}{2}\left(T_{i j} u^{i} u^{j}+\theta_{i j} q^{i} u^{j}-V_{i j} q^{i} q^{j}\right) .
$$

The first order Lagrangian $\mathcal{L}_{q u}$ is

$$
\mathcal{L}_{q u}=\frac{\partial \bar{L}_{q u}}{\partial u^{i}}\left(\dot{q}^{i}-u^{i}\right)+\bar{L}_{q u}
$$

or

$$
\begin{gathered}
\mathcal{L}_{q u}=\left(T_{i j} u^{j}-\frac{1}{2} \theta_{i j} q^{j}\right)\left(\dot{q}^{i}-u^{i}\right)+ \\
+\frac{1}{2}\left(T_{i j} u^{i} u^{j}+\theta_{i j} q^{i} u^{j}-V_{i j} q^{i} q^{j}\right) .
\end{gathered}
$$

The canonical momenta are

$$
p_{i}=\frac{\partial \mathcal{L}_{q u}}{\partial \dot{q}^{i}}=T_{i j} u^{j}-\frac{1}{2} \theta_{i j} q^{j},
$$

from which

$$
u^{i}=\left(T^{-1}\right)^{i j}\left(p_{j}+\frac{1}{2} \theta_{j k} q^{k}\right) .
$$

On the other hand, the Hamiltonian - expressed also in terms of $q^{i}$ and $u^{j}-$ is

$$
H_{q u}=\frac{1}{2}\left(T_{i j} u^{i} u^{j}+V_{i j} q^{i} q^{j}\right) .
$$

Now we can rewrite the first order Lagrangian $\mathcal{L}_{q u}$ (in a Palatini like fashion) as

$$
\mathcal{L}_{q u}=p_{i} \dot{q}^{i}-H,
$$

with (16) and (18) in (19) we get

$$
\mathcal{L}_{q u}=\left(T_{i j} u^{j}-\frac{1}{2} \theta_{i j} q^{j}\right) \frac{d q^{i}}{d t}-\frac{1}{2}\left(T_{i j} u^{i} u^{j}+V_{i j} q^{i} q^{j}\right)
$$

Varying $q$ and $u$ independently, the following first order equations of motion are found

$$
\begin{aligned}
\frac{d}{d t}\left(T_{i j} u^{j}-\frac{1}{2} \theta_{i j} q^{j}\right)-\frac{1}{2} \theta_{i j} \dot{q}^{j}+V_{i j} q^{j} & =0, \\
T_{i j}\left(\dot{q}^{j}-u^{j}\right) & =0 .
\end{aligned}
$$

which are equivalent to the original equations (12) (provided $\operatorname{det} T_{i j} \neq 0$ ) plus the definition of the variables $u^{j}$.

A different set of variables may be used for the Lagrangian and Poisson brackets relations. Let us choose $p_{k}$ variables defined in (16). That means that

$$
\dot{q}^{i}=\left(T^{-1}\right)^{i j}\left(p_{j}+\frac{1}{2} \theta_{j k} q^{k}\right),
$$

and therefore the Palatini like Lagrangian $p_{i} \dot{q}^{i}-H$ reads now

$$
\begin{aligned}
\mathcal{L}_{q p} & =p_{i} \dot{q}^{i}-\frac{1}{2}\left[\left(p_{i}+\frac{1}{2} \theta_{i k} q^{k}\right)\left(T^{-1}\right)^{i j}\left(p_{j}+\frac{1}{2} \theta_{j m} q^{m}\right)+\right. \\
& \left.+V_{i j} q^{i} q^{j}\right] .
\end{aligned}
$$

The first order equations of motion (varying $q$ and $p$ independently) turn out to be

$$
\begin{aligned}
\dot{p}_{i}-\frac{1}{2} \theta_{i j}\left(T^{-1}\right)^{j k}\left(p_{k}+\frac{1}{2} \theta_{k m} q^{m}\right) & =0, \\
\dot{q}^{i}-\left(T^{-1}\right)^{i j}\left(p_{j}+\frac{1}{2} \theta_{j m} q^{m}\right) & =0,
\end{aligned}
$$

which are, of course, equivalent to (12).

In summary, we have a second order Lagrangian $\left(L_{q \dot{q}}\right.$ in (11)) which gives rise to the second order equations of 
motion (12), and two first order Lagrangians $\left(\mathcal{L}_{q u}\right.$ in (20) and $\mathcal{L}_{q p}$ in (22) ) which give rise to first order equations which are equivalent to the second order ones.

The two different sets of variables $\left\{q^{i}, u^{j}\right\}$ and $\left\{q^{i}, p_{j}\right\}$ have, of course, different Poisson Bracket relations. In fact, compute the Lagrange bracket $\sigma_{a b}$ for (20), as well as its inverse (up to a sign) $\mathbf{J}^{a b}$

$\sigma_{a b}=\left(\begin{array}{cc}\theta_{i j} & T_{i j} \\ -T_{i j} & 0\end{array}\right), \quad \mathbf{J}^{a b}=\left(\begin{array}{cc}0 & \left(T^{-1}\right)^{i j} \\ -\left(T^{-1}\right)^{i j} & -\left(T^{-1} \theta T^{-1}\right)^{i j}\end{array}\right)$

where $\{i, j\} \in\{1,2, \cdots, n\}$ and the coordinates of the phase space are $x^{a}=\left\{q^{1}, q^{2}, \cdots, q^{n}, u^{1}, u^{2}, \cdots, u^{n}\right\}$.

For the Lagrangian (22), instead, we have the canonical Lagrange bracket $\sigma$ and its inverse (up to a sign) J

$$
\sigma_{a b}=\left(\begin{array}{cc}
0 & \mathbb{1} \\
-\mathbb{1} & 0
\end{array}\right), \quad \mathbf{J}^{a b}=\left(\begin{array}{cc}
0 & \mathbb{1} \\
-\mathbb{1} & 0
\end{array}\right)
$$

In the next section we will exhibit three Hamiltonian systems which give rise to the same classical equations of motion. Two of them are constructed starting from the aforementioned first order Lagrangian structures. The third hamiltonian structure cannot be derived from a second order Lagrangian.

\section{CLASSICAL EQUATIONS OF MOTION AND HAMILTONIAN STRUCTURES}

A Hamiltonian structure is defined by (8), (9) and (7). Consider now the first set of variables $\{q, u\}$, used in the previous section. A Hamiltonian system is defined by the following Poisson bracket relations (24) and Hamiltonian function (18)

$$
\begin{gathered}
{\left[q^{i}, q^{j}\right]=0, \quad\left[u^{i}, u^{j}\right]=-\left(T^{-1}\right)^{i k} \theta_{k m}\left(T^{-1}\right)^{m j},} \\
{\left[q^{i}, u^{j}\right]=\left(T^{-1}\right)^{i j},} \\
H=\frac{1}{2}\left(T_{i j} u^{i} u^{j}+V_{i j} q^{i} q^{j}\right) .
\end{gathered}
$$

Hamilton's equations are equivalent to (23) and the second order ones are equivalent to our starting set (12).

For the system described in variables $\{q, p\}$, the Hamiltonian system is defined as follows

$$
\begin{aligned}
{\left[q^{i}, q^{j}\right] } & =0, \quad\left[p_{i}, p_{j}\right]=0, \quad\left[q^{i}, p_{j}\right]=\delta^{i}{ }_{j}, \\
H & =\frac{1}{2}\left(\left(p_{i}+\frac{1}{2} \theta_{i k} q^{k}\right)\left(T^{-1}\right)^{i j}\left(p_{j}+\frac{1}{2} \theta_{j m} q^{m}\right)+\right. \\
& \left.+V_{i j} q^{i} q^{j}\right) .
\end{aligned}
$$

for which second order equations of motion are again (12).

The preceding structures are two versions (using different phase space variables) of Hamiltonian theories derived from the second order Lagrangian (11).

The general results may now be applied to a special case $T_{i j}=V_{i j}$. In this case, we can construct a third
Hamiltonian structure. In fact, let us denote the $2 n$ coordinates of phase space by $\left\{q^{i}, v_{j}\right\}$. The following Poisson brackets and Hamiltonian function define a third Hamiltonian structure for (12)

$$
\begin{gathered}
{\left[q^{i}, v_{j}\right]=\delta^{i}{ }_{j}, \quad\left[v_{i}, v_{j}\right]=0,} \\
{\left[q^{i}, q^{j}\right]=\left(T^{-1}\right)^{i k} \theta_{k m}\left(T^{-1}\right)^{m j},} \\
H=\frac{1}{2}\left(\left(T^{-1}\right)^{i j} v_{i} v_{j}+T_{i j} q^{i} q^{j}\right) .
\end{gathered}
$$

Hamilton's equations turn out to be

$$
\begin{aligned}
& \dot{q}^{i}=\left(T^{-1}\right)^{i j} \theta_{j m} q^{m}+\left(T^{-1}\right)^{i j} v_{j}, \\
& \dot{v}_{i}=-T_{i j} q^{j},
\end{aligned}
$$

which are equivalent to (12) with $V=T$.

A first order Lagrangian for these equations of motion can be calculated directly from our discussion in Section II, namely

$$
\begin{aligned}
L_{q v} & =v_{k} \dot{q}^{k}+\frac{1}{2}\left(T^{-1} \theta T^{-1}\right)^{k m} v_{k} \dot{v}_{m}- \\
& -\frac{1}{2}\left(\left(T^{-1}\right)^{i j} v_{i} v_{j}+T_{i j} q^{i} q^{j}\right),
\end{aligned}
$$

and it is a straightforward matter to prove that first order equations of motion are

$$
\begin{aligned}
& \dot{q}^{i}=\left(T^{-1}\right)^{i k} v_{k}-\left(T^{-1} \theta T^{-1}\right)^{k m} \dot{v}_{m}, \\
& \dot{v}_{i}=-T_{i j} q^{j}
\end{aligned}
$$

It is worthwhile mentioning that this Hamiltonian structure is not derivable from a second order Lagrangian [6], 7] .

In the following section we will discuss a physical example where these three Hamiltonian structures are considered.

\section{LANDAU PROBLEM AND NONCOMMUTATIVE SPACES}

In this section we analyze two very well known systems which are special cases of the examples discussed above, namely, the charged particle in an external, constant magnetic field - particle which, upon quantization, originates the so called Landau Levels - and the noncommutative harmonic oscillator as treated by Nair and Polychronakos [19]. The first one corresponds to a system as the one described by variables $\{q, p\}$ or $\{q, u\}$, while the second one corresponds to a system in variables $\{q, v\}$.

\section{A. Symmetric Gauge}

Consider, then, a non relativistic particle with charge $e$ and mass $m$ in a region of constant magnetic field $\mathbf{B}$. In the symmetric gauge the magnetic vector potential is

$$
\mathbf{A}=-\frac{1}{2} \mathbf{r} \times \mathbf{B}
$$


with $\mathbf{B}=B \hat{z}$, ( $B$ constant $)$. The Lagrangian and the Hamiltonian of this system are very well known. We will only write the Hamiltonian which is useful for our discussion. The Hamiltonian turns out to be

$$
H=\frac{1}{2 m}\left(p_{1}+\frac{1}{2} e B q^{2}\right)^{2}+\frac{1}{2 m}\left(p_{2}-\frac{1}{2} e B q^{1}\right)^{2}+\frac{1}{2 m} p_{3}^{2},
$$

and the canonical Poisson brackets

$$
\left[q^{i}, q^{j}\right]=0, \quad\left[q^{i}, p_{j}\right]=\delta_{j}^{i}, \quad\left[p_{i}, p_{j}\right]=0 .
$$

Since $p_{3}$ is a conserved quantity, the problem can be reduced to two dimensions. This is just the Hamiltonian system described by equations (27) with

$$
T_{i j}=m \delta_{i j}, \quad \theta_{i j}=\epsilon_{i j} e B, \quad V_{i j}=0 .
$$

However, it is also well known [20], 21] that one can define a set of noncanonical phase space variables which give rise the same equations of motion. In concrete, consider the Hamiltonian system defined in terms of the Poisson Brackets relations of variables $\{q, u\}$ as follows

$$
\begin{array}{r}
{\left[q^{i}, q^{j}\right]=0, \quad\left[q^{i}, u_{j}\right]=\delta_{j}^{i}, \quad\left[u_{i}, u_{j}\right]=-e B \epsilon_{i j},} \\
{\left[u_{i}, u_{3}\right]=0, \quad H=\frac{m}{2}\left(u_{1}^{2}++u_{2}^{2}+u_{3}^{2}\right) .}
\end{array}
$$

Matrices $T, V, \theta$ are defined as before, so that the previous Hamiltonian system is equivalent to (26).

Clearly, both systems are not connected by canonical transformations and might, in principle, give rise to inequivalent quantum theories.

In what follows we discuss the noncommutative harmonic oscillator.

\section{B. Noncommutative harmonic oscillator}

In [19] the quantum mechanics of the harmonic oscillator in a fully noncommutative space, i.e, a space where coordinates commutators and momenta commutators do not vanish, has been discussed.

From the point of view of the present article, the starting point are the classical EoM of the system which we will write in terms of variables $\{q, u\}$

$$
\begin{aligned}
& \dot{q}^{i}=\delta^{i j} u_{j}, \\
& \dot{u}_{i}=\left(B+\theta \omega^{2}\right) \epsilon_{i}{ }^{j} u_{j}-(1-B \theta) \omega^{2} \delta_{i j} q^{j}
\end{aligned}
$$

with $\{i, j\} \in\{1,2\}$.

For this set of equations at least two Hamiltonian structures may be defined. One of them is the following

$$
\begin{array}{r}
{\left[q^{i}, q^{j}\right]=0, \quad\left[q^{i}, u_{j}\right]=\delta_{j}^{i}, \quad\left[u_{i}, u_{j}\right]=\left(B+\theta \omega^{2}\right) \epsilon_{i j}} \\
H_{1}=\frac{1}{2}\left(u_{1}^{2}+u_{2}^{2}\right)+\frac{\omega^{2}}{2}(1-B \theta)\left(q_{1}^{2}+q_{2}^{2}\right) .
\end{array}
$$

There is a second order Lagrangian from which these EoM can be derived as it can be readily seen by comparing them with the Hamiltonian structure (26). Note that $T_{i j} \neq V_{i j}$. Besides, $\operatorname{det}\left(\boldsymbol{J}_{1}\right)=1$ and therefore the Poisson Brackets matrix is nowhere singular.

Another Hamiltonian structure for the same set of EoM is the following (we preserve previous notation for comparison purposes)

$$
\begin{aligned}
{\left[q^{i}, q^{j}\right]=} & \theta \epsilon_{i j}, \quad\left[q^{i}, u_{j}\right]=\left(1+\theta^{2} \omega^{2}\right) \delta_{j}^{i}, \\
& {\left[u_{i}, u_{j}\right]=\left(\theta^{3} \omega^{4}+2 \theta \omega^{2}+B\right) \epsilon_{i j} } \\
H_{2}= & \frac{1}{2}\left(u_{1}^{2}+u_{2}^{2}\right)+\frac{\omega^{2}}{2}\left(1+\theta^{2} \omega^{2}\right)\left(\left(q^{1}\right)^{2}+\left(q^{2}\right)^{2}\right) \\
+ & \omega^{2} \theta\left(q^{1} u_{2}-q^{2} u_{1}\right) .
\end{aligned}
$$

In this case, one can check that the Poisson Bracket matrix has a singularity in parameter space for $\theta B=1$ since $\operatorname{det}\left(\mathbf{J}^{\mathrm{NC}}\right)=(1-\theta B)^{2}$.

For completeness, let us write the classical Hamiltonian structure which leads, upon quantization, to the noncommutative harmonic oscillator (using the notation of previous sections)

$$
\begin{aligned}
{\left[q^{i}, q^{j}\right] } & =\theta \epsilon_{i j}, \quad\left[q^{i}, v_{j}\right]=\delta_{j}^{i}, \quad\left[v_{i}, v_{j}\right]=B \epsilon_{i j} \\
H^{\mathrm{NC}} & =\frac{1}{2}\left(\left(v_{1}\right)^{2}+\left(v^{2}\right)^{2}+\omega^{2}\left(\left(q^{1}\right)^{2}+\left(q^{2}\right)^{2}\right)\right)
\end{aligned}
$$

Clearly, this Hamiltonian structure has $T_{i j}=V_{i j}$, up to time rescaling. There is no second order Lagrangian for this system because coordinates have non vanishing Poisson Brackets relations [6], [7]. Moreover, the Poisson Bracket structure is singular for $\theta B=1$.

In summary, the classical equations of motion under study can be derived from a Hamiltonian system which may be obtained from a second order Lagrangian or, from a Hamiltonian system which is not derivable from a second order Lagrangian because coordinates have non vanishing Poisson Brackets relations. In this last case, space turns out to be noncommutative after quantization.

In the next section we study the quantization of these systems.

\section{QUANTUM MECHANICS}

In this section we will calculate explicitly the energy levels of the systems previously discussed. For the case of Landau Levels, as well as the noncommutative harmonic oscillator, these results are very well known and we will limit ourselves just to show the results in order to compare with the ones obtained for the new cases.

\section{A. Landau Levels}

Quantization of (34), once restricted to the plane $p_{3}$ constant, gives rise to an energy spectrum known as Landau Levels. The energy levels of this Hamiltonian system 
are [21]

$$
E_{\ell}^{\text {Landau }}=\frac{p_{3}^{2}}{2 m}+\omega_{0}(\ell+1 / 2),
$$

with $\omega_{0}=e B / m, \hbar=1, c=1$ and $\ell=0,1,2 \cdots$.

The wave function is

$$
\psi_{\ell}(q) \propto e^{-\frac{m \omega}{2}\left(q^{2}-q_{0}^{2}\right) q^{2}} H_{\ell}\left(\sqrt{m \omega}\left(q^{2}-q_{0}^{2}\right)\right),
$$

where $q^{2}$ is one of the coordinates on the plane spanned by coordinates $\left(q^{1}, q^{2}\right), q_{0}^{2}$ is a constant and $H_{\ell}(q)$ is the Hermite polynomial of order $\ell$.

The quantum mechanics of Hamiltonian system (35) gives the same results as the usual case previously summarized. Indeed, it is enough to note that a realization of the commutators algebra is given by the usual coordinate basis $q^{1}, q^{2}$ and the following operators $u_{i}$

$$
u_{i}=-i \partial_{i}-e A_{i},
$$

which, once implemented into the Hamiltonian, reproduces the Hamiltonian in variables $\{q, p\}$.

Therefore, the two Hamiltonian systems, upon quantization give rise to the same quantum theory. This is a non trivial result because, even if both systems are related by a rather trivial relation such as (41), the transformation $\left\{q^{i}, p_{j}\right\} \leftrightarrow\left\{q^{i}, u_{j}\right\}$ is not canonical.

\section{B. Noncommutative harmonic oscillator}

Let us consider the Hamiltonian system (36). The algebra of commutators turns out to be

$$
\left[q^{i}, q^{j}\right]=0, \quad\left[q^{i}, u_{j}\right]=i \delta_{j}^{i}, \quad\left[u_{i}, u_{j}\right]=i\left(B+\theta \omega^{2}\right) \epsilon_{i j}
$$

which has a realization in coordinate representation $\left\{q^{1}, q^{2}\right\}$

$$
u_{i}=-i \nabla_{q^{i}}+\frac{1}{2}\left(B+\theta \omega^{2}\right) \epsilon_{i j} q^{j}
$$

By doing that, the Schrödinger equation of the system is

$$
\left[-\frac{1}{2} \nabla_{q}^{2}+\frac{1}{2} \Omega^{2} \mathbf{q}^{2}+\frac{i}{2} \lambda\left(q^{1} \partial_{2}-q^{2} \partial_{1}\right)\right] \psi(q)=E \psi(q)
$$

with

$$
\Omega^{2}=\omega^{2}+\frac{1}{4}\left(B-\theta \omega^{2}\right)^{2}, \quad \lambda=\left(B+\theta \omega^{2}\right) .
$$

This equation can be solved completely. In order to do that it is convenient to parameterize the coordinate space $\left\{q^{1}, q^{2}\right\}$ in polar coordinates $(r, \varphi)$. The normalizable wave function turns out to be

$$
\psi_{n}^{\ell}(r, \varphi)=e^{-\frac{\Omega^{2}}{2} r^{2}} e^{i \ell \varphi} L_{n}^{\ell}\left(\Omega r^{2}\right) .
$$

with $L_{n}^{\ell}$, the Legendre's Polynomials and $\ell, n=0,1, \cdots$. The Hamiltonian eigenvalues are

$$
E_{n, \ell}=\Omega(n+\ell+1)-\frac{\ell \lambda}{2} .
$$

This is different from the noncommutative harmonic oscillator spectrum, which is given in terms of the spectra of two harmonic oscillators with different frequencies.

Finally, let us discuss the quantum mechanics of $\mathrm{H}_{2}$. Consider the canonical coordinate representation. Let $x_{1}, x_{2}$ be the space coordinates and $p_{j}$ the canonical momenta, which in coordinate representation has the standard form $p_{j}=-i \partial_{x_{j}} \equiv-i \partial_{j}$. Then, a realization of the quantum commutators version of (37) is

$$
\begin{aligned}
& q^{1}=\sqrt{\theta} x_{1}, \quad q^{2}=\sqrt{\theta} p_{1}, \\
& u_{1}=\sqrt{\frac{\theta B-1}{\theta}} x_{2}+\frac{1+\theta^{2} \omega^{2}}{\sqrt{\theta}} p_{1} \\
& u_{2}=\sqrt{\frac{\theta B-1}{\theta}} p_{2}-\frac{1+\theta^{2} \omega^{2}}{\sqrt{\theta}} x_{1} .
\end{aligned}
$$

for $\theta>0$ and $B \theta>1$.

The Hamiltonian turns out to be

$$
\begin{aligned}
2 \theta H_{2} & =\left(1+\theta^{2} \omega^{2}\right)\left(p_{1}^{2}+x_{1}^{2}\right)+(\theta B-1)\left(p_{2}^{2}+x_{2}^{2}\right)- \\
& -4 \sqrt{\theta B-1} L_{3}
\end{aligned}
$$

with $L_{3}=x_{1} p_{2}-x_{2} p_{1}$.

This Hamiltonian corresponds to an anisotropic harmonic oscillator with an angular momentum term. Indeed, it has the following structure

$$
H=U\left(p_{1}^{2}+x_{1}^{2}\right)+V\left(p_{1}^{2}+x_{1}^{2}\right)+W L_{3}
$$

for $U, V, W$, constants, and it is always possible to put it in the form

$$
H=\frac{1}{2 M} \mathbf{p}^{2}+\frac{1}{2} M\left(\Omega_{1}^{2} x_{1}^{2}+\Omega_{2}^{2} x_{2}^{2}\right)+W L_{3}
$$

This is not a diagonalizable system as it was shown in [23], and therefore the quantum mechanics it describes, is different from the two cases before analyzed.

Furthermore, in this case the wave function cannot, even in principle, be written in terms of the coordinates (because they do not commute). There is no way to compare the results obtained for this quantum system with the one described by commuting coordinates.

\section{CONCLUSIONS AND OUTLOOK}

In this paper we deal with one classical set of second order equations of motion and we approach the construction of Hamiltonian structures in three different ways.

We start from a second order Lagrangian for the system under consideration and construct its Hamiltonian structure in the usual way using two different sets of 
phase space variables $\{q, p\}$ and $\{q, u(\equiv \dot{q})\}$. The Poisson brackets relations and the Hamiltonian functions for both sets of phase space coordinates are exhibited. The quantum theories are worked out and they turn out to be equivalent.

Nevertheless, when the oscillator is described in terms of non commuting coordinates, the quantum theory has a spectrum which is different from the one previously found. Furthermore, its wave functions cannot even be compared to the ones obtained when using commuting coordinates. In this context, see also [12].

Acknowledgements: This work was supported by grants from FONDECYT-Chile grant-1095106, 1100777.
[1] H. Helmholtz, J. für die Reine und Ungerwandte Mathematik, Berlin 100, 133 (1887).

[2] G. Darboux, Leçons sur la Theorie Generale des Surfaces, Third Part, (Gauthier Villars, Paris), pag. 53 (1891).

[3] J. Douglas, Trans. Am. Math. Soc., 50, 71 (1941).

[4] P. Havas, Bull. Am. Phys. Soc. 1, 337 (1956); ibid, Supp. Nuovo Cimento 5, 363 (1957), ibid, Rev. Mod. Phys., 36, 938 (1964), ibid, Acta Phys. Austriaca 38, 145 (1973).

[5] S. Hojman and L. F. Urrutia, J. Math. Phys. 22, 1896 (1981).

[6] S. Hojman, J. of Phys. A 17, 2399 (1984).

[7] S. A. Hojman and L. C. Shepley, J. Math. Phys. 32, 142 (1991).

[8] See e.g M. R. Douglas and N. Nekrasov, Rev. Mod. Phys. 73, 977 (2001).

[9] F. A. Asenjo and S. A. Hojman, arXiv:0906.1627 1 [math-ph]].

[10] S. Hojman, J. of Phys. A 17, 2399 (1984).

[11] Sergio A. Hojman, AIP Conference Proceedings 365, Latin American School of Physics XXX ELAF, Group Theory and Applications, 117 (1996).

[12] S. Hojman y R. Montemayor, Hadronic J. 3, 1644 (1980)

[13] I. Hinchliffe, N. Kersting, Y. L. Ma, Int. J. Mod. Phys. A19, 179-204 (2004).

[14] See e.g, A. H. Chamseddine, A. Connes, Phys. Rev. Lett. 77, 4868-4871 (1996).

[15] For more references see e.g. in, A. Das, H. Falomir, M. Nieto, J. Gamboa, F. Mendez, Phys. Rev. D84, 045002 (2011); H. Falomir, J. Gamboa, M. Loewe, J. C. Rojas, and F. Mendez, Phys. Rev. D85, 025009 (2012).

[16] Some additional references are: F. Delduc, Q. Duret, F. Gieres, M. Lefrancois, J. Phys. Conf. Ser. 103, 012020
(2008); P. D. Alvarez, J. Gomis, K. Kamimura and M. S. Plyushchay. Phys. Lett. B659, 906 (2008); A. Berard, H. Mohrbach, Phys. Lett. A352, 190 (2006); K. Bolonek and P. Kosinski, arXiv:0704.2538; A. Kijanka and P. Kosinski, Phys. Rev.D70, 127702 (2004); L. Mezincescu, hep-th/0007046]; C. Duval and P.A. Horvathy, Phys. Lett. B547, 306 (2002); C. Duval and P.A. Horvathy, Phys. Lett. B479, 284 (2000); D. V. Vassilevich, JHEP 0805 (2008) 093; M. Gomes and V. G. Kupriyanov, arXiv:0902.3252 [math-ph]; P. A. Horvathy, M. S. Plyushchay, JHEP 0206, 033 (2002); J. Gamboa, M. Loewe, J. C. Rojas, Phys. Rev. D64, 067901 (2001);G. V. Dunne, J. Jackiw and C. Trugenberger, Phys. Rev. D 41661 (1990).

[17] H. Falomir, J. Gamboa, J. Lopez-Sarrion, F. Mendez, P.A.G. Pisani, Phys. Lett. B680, 384 (2009); A. Das, J. Gamboa, F. Torres and F. Méndez, Phys. Lett. A 375, 1756-1759 (2011).

[18] H. Falomir, J. Gamboa, M. Loewe, M. Nieto, J. of Phys. 45135308 (20129, arXiv:1109.6666 [math-ph]].

[19] V. P. Nair, A. P. Polychronakos, Phys. Lett. B505, 267274 (2001). arXiv:hep-th/0011172 [hep-th]].

[20] R. E. Peierls, Proc. Roy. Soc. 214 , 143 (1952); see also [21].

[21] See for example L. D. Landau and E. M. Lifshitz, Quantum Mechanics, Vol. 3 of Course of Theoretical Physics. Elsevier Science, Ed. 2004.

[22] Sergio A Hojman, J. Phys. A: Math. Gen. 29, 667 (1996).

[23] J. Gamboa, M. Loewe, F. Mendez and J.C. Rojas, Int. J. Mod. Phys.A17, 2555 (2002). 